\title{
Supersonic exciton gratings: coherent inter-polariton scattering in semiconductor microcavities
}

Birkedal, Dan; Vadim, Lyssenko; Hvam, Jørn Märcher

Published in:

Summaries of Papers Presented at the Quantum Electronics and Laser Science Conference, 2002. QELS '02. Technical Digest.

Link to article, DOI:

10.1109/QELS.2002.1031395

Publication date:

2002

Document Version

Publisher's PDF, also known as Version of record

Link back to DTU Orbit

Citation (APA):

Birkedal, D., Vadim, L., \& Hvam, J. M. (2002). Supersonic exciton gratings: coherent inter-polariton scattering in semiconductor microcavities. In Summaries of Papers Presented at the Quantum Electronics and Laser Science Conference, 2002. QELS '02. Technical Digest. (pp. 260-261). IEEE.

https://doi.org/10.1109/QELS.2002.1031395

\section{General rights}

Copyright and moral rights for the publications made accessible in the public portal are retained by the authors and/or other copyright owners and it is a condition of accessing publications that users recognise and abide by the legal requirements associated with these rights.

- Users may download and print one copy of any publication from the public portal for the purpose of private study or research.

- You may not further distribute the material or use it for any profit-making activity or commercial gain

- You may freely distribute the URL identifying the publication in the public portal 

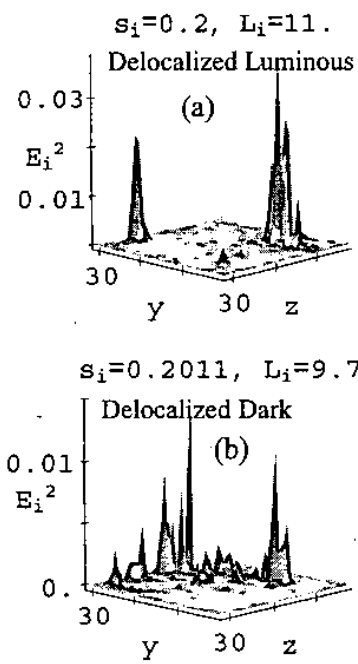

L $3 \mathrm{D}$ Space $8 \% \mathbf{\alpha 2} \% \mathbf{2}$

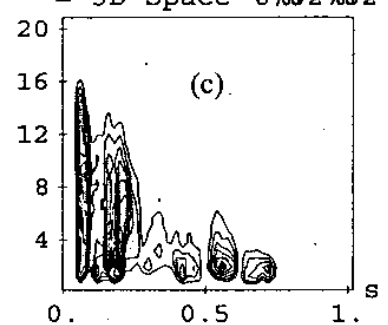

QFB6 Fig. 1. Examples of the delocalized luminous eigenmode (a) and delocalized dark eigenmode (b); the eigenvalues $s_{i}$ and localization (coherence) radii $L_{i}$ of the eigenmodes are shown. The distribution of the eigenmodes over their localization radius $L$ and spectral parameter $s$ is plotted as a contour map (c). Adapted from Ref. [1].

\section{References}

1. M.I. Stockman, S.V. Faleev, and D.J. Bergman, Phys. Rev. Lett., Vol. 87, 167401, 2001.

2. D.J. Bergman and D. Stroud, "Properties of Macroscopically Inhomogeneous Media", Solid State Phys., Vol. 46, 147, 1992.

\section{QFC}

8:00 am-9:45 am Room: 203

\section{Semiconductors: 1}

Steven T. Cundiff, NIST, USA, Presider

QFC1

8:00 am

Supersonic Exciton Gratings: Coherent Inter-polariton Scattering in Semiconductor Microcavities

D. Birkedal, V.G. Lyssenko, and J.M. Hvam, Research Center COM, Technical University of Denmark,Email: Birkedal@com.dtu.dk

Semiconductor microcavities (SMC) offers unique opportunities to reach physical condition

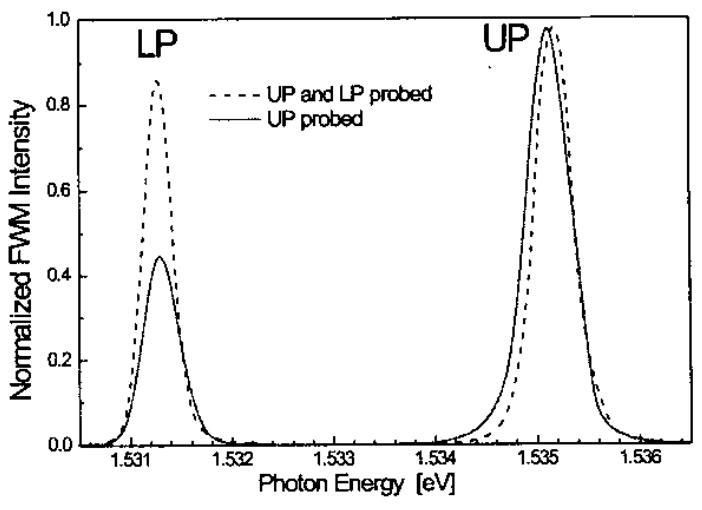

QFC1 Fig. 1. Spectrally resolved transient four-wave mixing at zero delay. Solid line for probing only the upper polariton (UP) and dashed line for probing simultaneously the upper and lower polariton (LP).

not easily attained otherwise. This has led to a range of interesting new effects, where in particular coherent scattering of polaritons have attracted considerably interest.' Coherent phenomena in semiconductor have most significantly been studied using transient four-wave mixing (TFWM), where in the case of e.g. quantum wells (QWs) a detailed understanding of the involved physics have resulted. ${ }^{2}$ In contrast, relatively few reports have been published on TFWM studies of SMC. ${ }^{3,4,5,6}$ These studies have shown that the coherent nonlinear dynamics of SMC polaritons is modified from that of e.g. heavy-hole-lighthole excitons in semiconductor QWs.

Here we report on a coherent nonlinear phenomenon in a $\mathrm{SMC}$, which has no parallel for QW excitons. When two different polariton modes of the SMC are impulsively excited they will undergo normal mode oscillations (NMOs) with coherent energy exchange between the exciton and the cavity mode. In our experiment the two polaritons are excited with slightly different angles resulting in a travelling wave exciton grating. When a test polariton mode is excited it will scatter in the travelling grating producing amplitude modulation sidebands. This phenomenon produces a TFWM signal, which is shifted in frequency from that of the test beam by the NMO frequency. In our case, in the $\mathrm{THz}$ range corresponding to a grating velocity of $\approx=1 \cdot 10^{7} \mathrm{~m} / \mathrm{s}$, which is four orders of magnitude larger than the sound velocity. The presence of such travelling exciton gratings have been predicted ${ }^{5}$ but so far not observed directly.

The sample under investigation is a GaAs/AlGaAs $\lambda$ cavity with a single $25 \mathrm{~nm} \mathrm{GaAs}$ QW at the center. ${ }^{7}$ The sample is held at $5 \mathrm{~K}$ in He cryostat and is excited by pulses from a self-modelocked Ti:Sapphire laser in the $2 \vec{k}_{2}-\vec{k}_{1}$ reflection geometry. The pulses are spectrally shaped to allow selective excitation of either of the polariton modes. The TFWM signal is detected spectrally resolved and recorded as a function of time delay between the two pulses. The present experiment are conducted for near resonant conditions.

First we excite $\left(\vec{k}_{1}\right)$ and probe $\left(\vec{k}_{2}\right)$ both polaritons with spectrally broad pulses. This results in TFWM signals on the upper and lower polariton as shown in the spectrum in Fig. 1. In addition, strong beats in the TFWM intensity is seen as a function of delay between the two pulses as

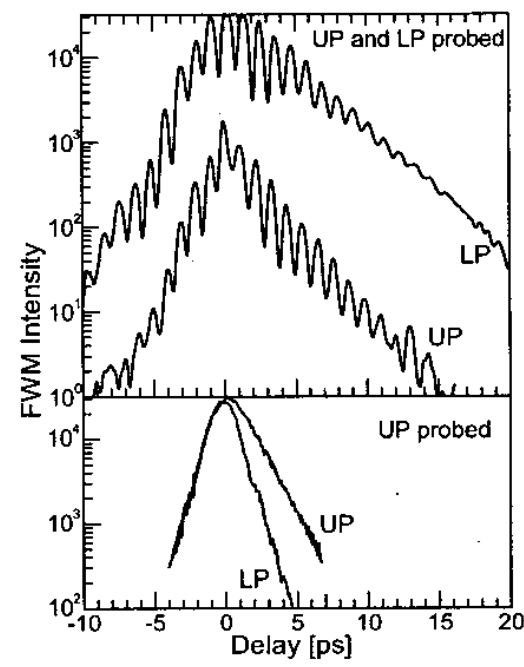

QFC1 Fig. 2. Transient four-wave mixing as a function of delay. Upper figure show the signals at the upper and lower polaritons (UP \& LP) when both polaritons are probed simultaneously. Lower figure for probing selectively the upper polariton.

shown in Fig. 2. This corresponds to our expectations from QW excitons and is in agreement with previous reports. In contrast, if we now probe selectively only one polariton, we observe a TFWM signal resonant with the other polariton. This is shown here for selective probing of the upper polariton [Fig. 1: TFWM spectrum; Fig. 2: delay dependence], where a strong signal resonant with the lower polariton is observed. We note that for this case, no beats and much faster decays of the TFWM intensity are observed [Fig. 2]. We will demonstrate that these observations are in agreement with a theory based on the combined Maxwell and optical Bloch equations. ${ }^{5,6,8}$

\section{References}

1. P.G. Savvidis et al., Phys. Rev. Lett. 84, 1547 (2000).

2. See e.g. J. Shah, Ultrafast Spectroscopy of Semiconductors and Semiconductor Nanostructures, (Springer, Berlin, 1999). 
3. H. Wang et al., Phys. Rev. B 51, 14713 (1995)

4. M. Kuwata-Gonokami et al., Phys. Rev. Lett. 79, 1341 (1997).

5. H. Wang et al., Phys. Rev. Lett. 81, 3255 (1998).

6. M. Koch, J. Shah, and T. Meier, Phys. Rev. B 57, R2049 (1998).

7. For details on the sample see e.g. J.R. Jensen, P. Borri, W. Langbein, and J.M. Hvam, Appl. Phys. Lett. 76, 3262 (2000).

8. See also H.J. Kimble; H.J. Carmichael et al., in Cavity Quantum Electrodynamics, edited by P.R. Berman (Academic, Boston, 1994).

\section{QFC2}

8:15 am

Coherent Combined Intersubband-cyclotron Resonances

J. Bao, R. Merlin, Department of Physics, The University of Michigan, Ann Arbor, Michigan, 48109-1120,Email:jmbao@umich.edu, merlin@umich.edu

L.N. Pfeiffer, K.W. West, Bell Laboratories, Lucent Technologies, Murray Hill, New Jersey, 07974

In two-dimensional (2D) electron systems, tilted magnetic fields lead to subband-Landau-level coupling and associated excitations of mixed character referred to as combined intersubbandcyclotron resonances. 'These hybrid modes have been extensively studied using infrared ${ }^{2}$ and Raman spectroscopy. ${ }^{3}$ Recently, the observation of coherent intersubband charge-density oscillations generated by ultrafast optical pulses was reported in a high mobility $2 \mathrm{D}$ electron gas. ${ }^{4}$ Here, we present results in the presence of a tilted magnetic field revealing coherent intersubband-cyclotron coupled oscillations.

Our sample is a 400 - $\AA$ one-sided modulationdoped GaAs-AlGaAs single quantum-well grown by molecular beam epitaxy. The top $\mathrm{Al}_{0.2} \mathrm{Ga}_{0.8} \mathrm{As}$ layer contains $\mathrm{Si}$ donors separated from the GaAs quantum well by a thick undoped space layer. The areal density of the electron gas at the interface is $\rho \approx 1.88 \times 10^{11} \mathrm{~cm}^{-2}$ and the low-temperature mobility is $2.88 \times 10^{6} \mathrm{~cm}^{2} / \mathrm{Vs}$. Pump-probe experiments were performed at $10 \mathrm{~K}$ with a modelocked Ti-sapphire laser giving $\sim 50 \mathrm{fs}$ pulses at a repetition rate of $82 \mathrm{MHz}$. The field is provided by a split coil superconducting magnet. The laser beams were focused onto a relatively large 0.3 $\mathrm{mm}$ diameter spot to reduce heating. The average power of the probe beam was $1 \mathrm{~mW}$ (the energy density per pulse is $U=2 \times 10^{-8} \mathrm{~J} / \mathrm{cm}^{2}$ ). The cen tral wavelength of the pulses was tuned to resonate with excitons associated with heavy-hole states at $\sim 806 \mathrm{~nm}$.

Time-domain results are shown in Fig. 1 for two different values of the magnetic field at a tilt angle $\theta \sim 47^{\circ}(\theta$ is the angle between the field and the normal to the sample surface). Both traces show two, a short- and a long-lived oscillation The magnetic-field dependence of the coupledmode frequencies is plotted in Fig. 2 together with data obtained from spontaneous Raman scattering measurements. As $B \rightarrow 0$, the higherfrequency branch approaches the bare intersubband charge-density mode whereas the frequency of the other branch goes to zero. Based on this behavior, the two branches are assigned to coupled intersubband-cyclotron modes. This assignment is supported by the comparison of the experi-

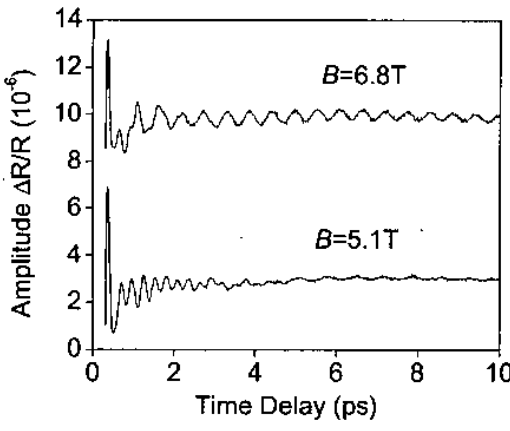

QFC3

Invited

8:30 am

Ultrafast Formation of Quasiparticles in Semiconductors: How Bare Charges Get Dressed

A. Leitenstorfer, M. Betz, A. Brodschelm, R. Huber, and F. Tauser, Physik-Department E11, TU München, James-Franck-Stra\$dse, D-85748 Garching, Germany, Email: aleitens@ph.tum.de

The quasi-particle model is one of the fundamental concepts in many-body physics. It is usually too complicated to regard each isolated ("bare") particle and to take into account its interactions with all the other individual components of the system. More physical insight is gained by introducing new units which are composed of the bare particle plus some average surrounding. Usually, these quasi-particles are assumed to form instantly. However, this picture turns out to be valid only on timescales which are long compared to the oscillation cycle of the collective mode of the system. Very recently, the quantum dynamical phenomena which occur during the formation of interparticle correlations in systems far from thermal equilibrium have become accessible.

As a first example, the dynamics of an isolated charge coupled to a highly polar crystal lattice via the Fröhlich interaction is discussed. After femtosecond excitation of heavy holes in CdTe, we observe a strong broadening of the energy distribution within $300 \mathrm{fs}$, even if the carriers are injected with an excess energy less than the LO phonon energy. This finding is in strong contrast to analogous measurements in weakly polar GaAs, where an energetically sharp distribution is found for the same conditions of small excitation densities of $5 \times 10^{14} \mathrm{~cm}^{-3}$ and low temperatures of $4 \mathrm{~K}$. A quantum kinetic description is necessary to explain the broadening of the hole distribution in CdTe: In the regime of intermediate electronphonon coupling, the polaron self-energy becomes comparable to the LO phonon energy. As a result, the virtual phonons which are generated due to deformation of the crystal lattice during transition from the bare charge to a polaron lead to significant changes in the carrier distribution functions. Energy conservation holds only within the coupled carrier-phonon system but not for the electronic excitations alone.

The second part of the contribution deals with carrier-carrier interactions: The ultrafast buildup of Coulomb screening and collective behavior in a dense electron-hole plasma photogenerated within $10 \mathrm{fs}$ are demonstrated. In the previous example of polaron formation, the dynamics of the carrier distribution had been monitored which is a result of a coupling process between elementary excitations. Ultrabroadband two-dimensional $\mathrm{THz}$ studies add a new dimension: direct access to the details of the interaction process itself. ${ }^{2}$ This is achieved by probing the dielectric response of the non-equilibrium system in the frequency regime of its collective mode, in our case a plasma resonance at $15 \mathrm{THz}$. It turns out that shortly after generation of the plasma, the amplitude and phase distortion of a single-cycle probe transient at $28 \mathrm{THz}$ is almost instantaneous. If the system is probed approximately $100 \mathrm{fs}$ after its excitation, we find a delayed response due to plasma oscillations. These observations are consistent with an ultrafast transition from an undressed state of bare charges far from equilibrium into a corre- 